\title{
Injuries of the heart and great vessels due to pins and needles
}

\author{
DAVID CHAS. SCHECHTER AND LAWRENCE GILBERT \\ From the Section of Cardiothoracic Surgery, Newark Beth Israel Hospital, Newark, N.J., U.S.A.
}

\begin{abstract}
Three instances of cardiac injury from needles in two adults are described. Trauma was accidental in one and due to suicidal attempts in the other two. The objects were removed. There are 157 published accounts of wounding of the heart and/or great vessels by pins and needles. The victims have ranged from infants to the elderly. Causative agencies were accidents, suicide, and homicide. A few were discovered at necropsy in presumably asymptomatic persons. Six of the accidental injuries were iatrogenic. The objects reached the heart or great vessels from transthoracic insertion, ingestion, embolization, aspiration, or transabdominal penetration. The overall mortality incidence was about $50 \%$. Acute cardiac tamponade was the dominant cause of death. Almost all individuals survived who were operated upon and from whom the object was removed. The right ventricle was hurt most often, but no region of the heart or of the great vessels was spared. Occasionally multiple parts were affected. The primary damage occurred principally while the foreign body was extracardiac and relatively immobilized, from repetitive scratching or puncturing of the beating heart. Chest pain and unfolding patterns of tamponade were inconsistent in onset, severity, and duration. Death ensuing days or weeks after the initial injury was frequent. Progressive haemopericardium in some cases was due to or aggravated by laceration of a structure from within outward. Late complications-several fatal-were consequent upon inflammation, sepsis or thromboembolism. It is urged that all foreign bodies in the heart or great vessels be retrieved, even if seemingly innocuous clinically.
\end{abstract}

'One discovers the heart under the unexpected aspect of a bric-a-brac shop, where may be found objects as disparate as in the fabled stomach of the ostrich.' Dr. Chas. Esmein (1910)

Cardiac wounding by foreign bodies poses problems in management that are dissimilar from those of other pathological conditions. That the heart or great vessels may be severely damaged by objects of unimpressive size or appearance is a fact. In the main, preoccupation with this variety of injury has pertained to bullets, shrapnel, and kindred projectiles. However, similar trauma has also occurred in consequence of strikingly diversified items. A pot-pourri of objects has been inculpated: the spine of a sting fish, metallic splinters, tooth picks, dental plates, fish bones, pieces of ice-pick blades, bone spicules or sequestra, scraps or rods of wood, glass slivers, an aluminium pipe stem, thorns, stud gun nails, metal files, drill fragments, fruit pips, vegetable fibres, an orthopaedic wire strut, a rubber catheter, a metal IPresent address: Section of Cardiovascular and Thoracic Surgery, New York Medical College, New York, N.Y. cannula, a false tooth, a straw from a broom, ando a worm (Bláha, 1935 ; Decker, 1939 ; Barrett 1950 ; Lowen, Fink, and Helpern, 1950 ; Bajardio 1953 ; Tänzer, 1953 ; Farber and Craig, 1956 Parmley, Mattingly, and Manion, 1958 ; Weekleyo and Maltby, 1963 ; Gajdas, 1963 ; Leonard and Gifford, 1965 ; Hudson, 1965).

Over the past decade numerous accounts haver been published of iatrogenic mishaps resulting in pieces of diverse medical paraphernalia being swept into the heart or great vessels. Disconcerting situations have arisen from the breakage and losso into the blood stream of venoclysis tubing, Pudenz-Holter valves for decompression in hydro응 cephalus, and flexible steel stylets used duringe cardiac catheterization (Lillehei, Bonnabeau, andळ Grossling, 1965).

Wounds of the heart and great vessels due to pins and needles are not common: nor are theyo harmless. This communication reports a personaf experience with three instances of sewing needle injuries to the heart in two patients. 


\section{CASE REPORTS}

\section{CASE 1}

First admission B. B., a 29-year-old woman, was first seen by one of us (L. G.) in December 1960 at her psychiatrist's consulting-room. On the preceding evening, while sitting, she had partly pushed an ordinary sewing needle into the front of her left chest. The pain made her stand up, and with this sudden movement the remainder of the needle disappeared beneath the skin. The pain was relieved when she lay down in the prone position.

There was a minute fresh puncture site in the 5th intercostal space just left of the sternum. Fluoroscopy showed the needle to be entirely within the cardiac silhouette and moving synchronously with each beat. The deepest portion of the needle moved more vigorously than its superficial parts.

Operation was performed approximately 18 hours after injury. Thoracotomy was through a submammary 5th intercostal incision. The hub of the needle was palpated at a small ecchymotic area in the pericardial fat over the right ventricle. On dissecting the fat a glint of metal was perceived. The needle was found still projecting through the pericardium. It was grasped with a haemostat and withdrawn gently. Oozing of dark blood through the wound in the pericardium followed, and the latter was opened widely anterior to the phrenic nerve. Approximately $50 \mathrm{ml}$. of blood was aspirated. The pericardial sac was left open.

The patient's recovery was uncomplicated. Radiological studies on the seventh postoperative day showed a normal cardiac silhouette, no haemothorax, and no other foreign bodies in the chest wall or cavity. On this same day the patient was transferred to an institution for psychiatric care.

One week later the patient's physician announced that chest radiographs taken that day had disclosed two foreign bodies. These films revealed a sewing needle located in the lateral lung field, possibly in the costophrenic sinus. A second, partially curved, broken needle was deep in the subcutaneous tissue, anterior to the sternum. The consensus was to leave these objects in place.

Second admission In December 1965, B. B. (now 34 years of age) was referred directly from the psychiatrist's consulting-room after remarking that she had plunged a needle into her chest five days previously during a fit of mental depression. Radiography and fluoroscopic examination showed the needle entirely within the cardiac silhouette and moving synchronously with each beat. In addition, the presence of two other foreign bodies, noted in the radiographs taken five years previously, was confirmed. Neither of these foreign bodies had migrated.

That same evening the left thorax was re-explored through the former incision. The pericardium was densely fused to both the heart and retrosternal tissues. Over the anterior surface of the right ventricle, an area of recent ecchymosis was seen; and soon, after dissection and search, about $1 \mathrm{~mm}$. of the needle hub was found projecting free of the adherent pericardium. The needle was easily withdrawn. There was no bleeding from the puncture site. Exploration of the subcutaneous tissue exposed the 'old' needle fragment sequestered there. Probing in the costophrenic sulcus, hampered by considerable adhesions, did not reveal the needle anticipated in this area. As it was considered to be harmless, no further attempts were made to retrieve it.

The patient's recovery was uncomplicated, and she was discharged on the eighth postoperative day to another institution for psychiatric treatment.

CASE 2 J. U., a 43-year-old roofer in the habit of carrying a needle in his left shirt pocket for removing splinters from his fingers, was knocked on the chest by a ladder. Sharp pain was experienced over the left breast, but this grew less within an hour, and he was able to resume work with no further discomfort. He noticed a speck of clotted blood on his undershirt and was aware of the loss of his needle, but was unconcerned about this until three days later, when again he received a glancing blow on the chest, this time from a swinging door. Pain ensued and persisted. It was sharp, localized to the left breast, and not altered by position or breathing.

Physical examination was normal; but a hard, tender, subcutaneous nodule, $0.75 \mathrm{~cm}$. in size, was palpable in the 5th intercostal space immediately lateral to the left of the sternum. The electrocardiogram was normal.

Radiography revealed a linear density in the region of the cardiac ventricular septum, its outer end directed towards the anterior chest wall, $4 \mathrm{~cm}$. from the skin. By fluoroscopy the free end of the needle was seen to vibrate synchronously with the heart beat.

Operation was undertaken about eight hours after admission. A left 5 th intercostal anterolateral incision was made and subsequently extended to the right, transsternally, without entering the right pleural cavity. No track was visible in the intercostal tissues or on the surface of the pericardium. Nor was there any imprint on the heart itself to indicate the entry of the needle. The pericardial sac contained less than $50 \mathrm{ml}$. of blood.

Methodical palpation and pressure on different parts of the heart failed to uncover the site of nidation of the needle. Recourse to an electromagnetic locator was not fruitful, and neither was probing of the right ventricular chamber with a haemostat inserted through its apex.

A finger was introduced into the right atrium, through the appendage, and about $1 \mathrm{~cm}$. of the point of the needle was felt immediately above the medial cusp of the tricuspid valve. By combined pressure on the needle and quickly flattening the heart in the anteroposterior plane by manual pressure it was possible to extrude the heel of the needle through 
the anterior cardiac wall and to grasp it with a haemostat and remove it. The pericardial sac was left open.

There were no arrhythmias. The patient withstood the procedure well, and there were no untoward postoperative sequelae from the manipulations described.

\section{ANALYSIS OF THE WORLD LITERATURE}

Cardiac injuries from pins are almost identical with those from needles. Some 157 cases of wounds caused by these objects have been collected up to 1967. They, together with those we have described, comprise the context of our review. Recitals of the modes of injury, as well as their nature, furnish rather provocative reading matter.

HISTORICAL The earliest documentation on this subject was made in 1600 by Paulus Zacchias, the father of forensic medicine. A priest decided on immolation by castrating himself and thrusting several stout needles into his body, including one through the heart. He survived for six days. Zacchias was duly impressed by this-'. . hic quidem mirabile auditu est . . .-for had not Aristotle himself stated that touching the heart brought about instantaneous death? In 1782, a Sardinian nobleman, at the court of King Amadeos, was slain during sleep by his exasperated wife. She plunged into his heart a needle which was made of gold. Admiral Pierre Charles JeanBaptiste Silvestre, the Duke of Villeneuve, being responsible for tactical blunders at the Battles of the Nile and Trafalgar, fell into disfavour with Napoleon. Despondent, he purchased anatomical plates to become acquainted with the arrangement of the thoracic viscera. Then, with precision, he passed a needle into his heart. He survived several years and was captured by the English on 22 April 1806.

It is surprising, considering the antiquity of cardiac acupuncture and its widespread use even in eastern. European countries during the cholera epidemics of the last century, that only a single death is on record from this type of injury (Schiff, 1965).

OBJECTS There are reports of 147 injuries from needles. Most were from plain household sewing or darning needles. In nine instances, two or more needles were used. In four, knitting needles of various lengths were incriminated. In one, a 9-cm. needle for mending sails was used in a suicide attempt. The shortest needle resulting in a fatality was less than $2 \mathrm{~cm}$. long. A gramophone needle was discovered in a heart at necropsy. Five cases were due to thoracentesis or to hypodermic needles? breaking at the hub. One involved embolism of a radium needle from the skin to the pulmonary artery.

The rest of the injuries were caused by assortects pins. The shortest which resulted in death was less? than $2 \mathrm{~cm}$. long; the longest was an 8-in. hatpin There were also a shawl pin, a brooch pin, twow hairpins, and four safety pins. Multiple pins weres used for suicidal purposes in two instances, by being swallowed or thrust into the body. A bluni 'bobby pin' was ingested accidentally and camoi to rest in the heart in one case.

CAUSATION The pins and needles entered the hearf muscle or its cavities chiefly by transthoracic penetration or by ingestion. In 88 authenticated case the injury was accidental. The transthoracic rout accounted for 68 of these, the invariable mechan? ism being a fall, a blow or an embrace, with theg object stuck originally in the victim's or someonee else's clothes. In one instance a man nearly died when a needle in the bodice of a barmaid with whom he was engaged in coitus traversed his chese wall. Six of the accidents were iatrogenic. The loss of a radium needle on that basis has pref viously been cited. The others were from break age of a needle during thoracentesis, intracardiae injection, and intravascular embolization during performance of venoclysis. In one child, the needle entered the thorax across the abdominal wall, and only in a single case was it aspirated. In the remainder, the object was swallowed.

There were 52 injuries consequent on suicide of suicidal design. All but seven, in which the objects. were swallowed, were transthoracic wounds. If some of these there were concomitant self-destruc 3 tive actions, such as insertion of glass into the vagina, amputation of genitalia, slashing the throat with a razor, drinking laudanum, and cutting the wrist. One woman swallowed an entire packet of needles. Another, to avoid drawing attentions feigned prayer, meanwhile surreptitiously pressing 30 pins and needles into her chest with a Biblon until haemorrhage from the superior vena cavă ended her life. One woman both swallowed needles and thrust several more into diverse parts of hef body.

Pins and needles were used as weapons for murder on five occasions. The most macabre incie dent concerned a neonate who was mutilate $\vec{\phi}$ almost beyond recognition by being stabbed $20^{\circ}$ times with a knitting needle and 40 times with 8 pen-knife. 
In six cases, the object was discovered fortuitously at necropsy. The mechanism of injury was not identified in the last nine.

AGE AND SEX There were 76 males and 60 females in the series. The sex of the other 24 was not stated. The youngest victim was the infant just mentioned ; two patients, the oldest, were 82 years of age.

As might be anticipated, all the infants and children were hurt accidentally, either in a fall or by being clasped against the bosom of a mother or nurse whose frock contained the object. About 45 of the accidental injuries took place in persons over the age of 20 years, the oldest being 82 years. The proportion of accidents in males was 43 to 35 in females. The sex of the remaining 10 accident victims was not stated.

In the suicidal group of 52 cases, the youngest was a 17-year-old adolescent, and the oldest was 82 years of age. There were 25 men and 22 women. The sex of the five others was not stated.

PHYSICAL SIGNS The quality, onset, duration, and temporal relationship of the symptoms varied widely. The most consistent was precordial or retrosternal pain. This was sharp, lancinating or dull. It was clamant uniformly at the start, becoming degravescent after irregular periods, and often recurring with different qualitative characteristics as a terminal event. Deep-seated pain and occasionally dysphagia were early but temporary manifestations of swallowed foreign bodies. The type of secondary pain arising after a relatively symptom-free interval, and described as an 'angoisse' or oppressive in intensity, is recognizable as characteristic of cardiac compression from haemopericardium. The severity of the initial pain was greater when the object was still extracardiac, as in the pericardium or soft tissues of the chest wall. The pain usually disappeared after complete entry of the object into the heart.

Dyspnoea preponderated next. It usually developed later and in association with increasing pericardial effusion. When it was pronounced, a sense of suffocation was complained of. Palpitations were rare.

Very early, the foreign bodies were either visible or palpable in the skin, depending on the force with which they had been driven in. Needles either left no trace of puncture on the skin or a small area of ecchymosis. The ingress of pins was ordinarily halted by their broadened end, but this was inconsistent. When there was a subcutaneous protrusion, it throbbed rhythmically with the heart beat. Cessation of this movement was usually an ominous sign, inferring that relay of the cardiac pulsations was stilled because of an interposed enlarging mass of blood. A cardiac murmur or pericardial friction rub was audible in a few patients.

Incidental symptoms such as retching, vomiting, weakness, cough, and syncope were ordinarily nonspecific accompaniments of trauma. Fever, sweats, and tachycardia usually indicated suppurative pericarditis, carditis or pleuritis.

Diagnosis was difficult in many of the infants and children, especially when information about trauma was vague or wanting. Several displayed unexplained lassitude, restlessness or anorexia. Others were given to recrudescent syncope or convulsions. A few showed cardiac decompensation.

In both adults and the young, ingravescence or dissolution followed an abrupt pattern, with sudden death or a rapid sequence of heart failure, jugular venous distension, shock, and coma presaging death from acute cardiac tamponade. This took place both early and late after injury.

DIAGNOSIS Immediate death has been uncommon. In most, the correct diagnosis was made, or was at least attainable, within a few hours from the precipitating trauma. On 10 occasions it was obvious, the article being seen transfixing the skin. In 14 others, the object was subcutaneous, and palpable, usually with transmitted cardiac pulsations.

When information about injury was volunteered by the patient or by an interested party, leading to speedy medical evaluation, the chief diagnostic aid was radiology. The shadow cast by a needle was unmistakable, and its intimate relationship to the heart was corroborated by its oscillations being visualized with fluoroscopy. Cardiac arrhythmias were rare, save as a terminal event ; electrocardiography was generally not helpful except to corroborate clinical suspicion of cardiac tamponade or pericarditis.

Diagnostic perplexities were presented by those infants regarding whom there was no foreknowledge of injury. The customary picture in these cases was one of mystifying physical deterioration lasting for days or weeks, culminating in frank cardiac tamponade. Often, without antecedent clinical portents, children became gravely ill and were admitted to hospital, after which an intracardiac foreign body was discovered by radiography.

Two people, a young boy and a middle-aged woman, insisted that they had a needle in the heart; neither was believed and both died. 
MANAGEMENT The object was removed with the fingers nine times out of the 10 that it jutted above the skin. In one case a needle broke when a child tried to take it out, and in another, the manipulation resulted in its being pushed further into the chest, necessitating thoracotomy. Death occurred in three of the cases when the object was pulled out with the fingers.

The foreign body was subcutaneous in 14 cases, once with an attached cotton thread trailing over the skin. In 11 of these, the object was removed after incising the skin over it. In three others these manœuvres were unsuccessful, and major operation followed. One patient died after removal of the object from the subcutaneous tissues.

Mediastinotomy, pericardiotomy, or formal thoracotomy, each of varying magnitude, was undertaken 53 times, and extraction by oesophagoscopy once. Six of these operations failed, the foreign body being left behind. Four other patients in whom emergency thoracotomy was undertaken died on the operating table or soon after.

In three cases, the object was extruded spontaneously by the heart. In one, it appeared after 10 days beneath the ensiform cartilage, and in a second, at a superior intercostal space after three weeks. Both were simply removed by incising the skin over them. In the third, a needle which had travelled from the antecubital vein to the left lung via the ipsilateral pulmonary artery over a period of eight years was removed by wedge resection of the lung.

FINDINGS AT OPERATION OR NECROPSY The affected anatomical structures were distributed thus:

\begin{tabular}{lrlr} 
Right ventricle & 66 & Left ventricle & 47 \\
Haemopericardium & 46 & Oesophagus & 4 \\
Focal or diffuse & & & 4 \\
$\quad$ pericarditis & 20 & Stomach & 4 \\
Ventricular septum & 16 & Tricuspid valve & 3 \\
Right atrium & 12 & Pulmonary artery & 4 \\
Lung & 11 & Aorta & 4 \\
Intracavitary & & & 4 \\
$\quad$ thrombus & 8 & Haemothorax & 4 \\
Left atrium & 7 & Mitral valve & 3 \\
Both ventricles & 7 & Superior vena cava & 2 \\
Papillary muscles & 7 & Pulmonary infarction & 2 \\
Diaphragm & 6 & Bronchus & 2 \\
Pleuritis & 6 & Vertebrae & 2 \\
Coronary vessels & 6 & Inferior vena cava & 1 \\
Mediastinitis or media- & & \\
\multicolumn{2}{r|}{ stinal haematoma 5} & Liver & 1
\end{tabular}

Not infrequently, the foreign body caused? damage by wounding adjacent structures, eithe by perforating them individually in transit or bs impaling them concomitantly 'en brochette'.

FATE Apart from the cases discovered coincio dentally at necropsy, and those patients who died. from unrelated or intercurrent disease, the mor? tality in the series was 85 cases, or roughly $50 \% \overrightarrow{\dot{\vec{\omega}}}$

Thirteen of the 85 deaths followed some form of major surgical intervention. Infection waș responsible for three, iatrogenic errors for twow acute tamponade for three, the moribund state of the patients at operation for four, and an inade quate procedure for one. Closer scrutiny of thछ causes of these deaths disclosed that, except as regards the individuals who were operated upon while desperately ill, all the fatalities could have been prevented. Thus, one of the iatrogenic mis chances consisted of laceration of a coronary ves? sel. In none of the three who developed tamponad had drainage of the pericardium been effectedo the surgeons being lulled into false security by the absence of demonstrable haemopericardium whew the object was removed.

The foreign body was taken out by some surgical means, major or minor, in 51 of the $5 \%$ survivors, and left in the heart in five. Of the latter, two died from unrelated ailments after $1 \overrightarrow{8}$ months, the third was reported to be in goo health a few years later, the fourth was barel salvaged after incurring empyema and suppurative pericarditis, and in the last the needle wast extruded eventually from the heart. Another per? son, from whom a needle was withdrawn but the pericardium was sutured, suffered in the pose operative period from pleuritis, pericarditis. cardiac arrhythmias, and spontaneous drainage through the wound of bloody pericardial fluid.

\section{DISCUSSION}

Foreign bodies may reach the heart by any of the following pathways: through the chest wallo directly through the sternum, ribs, or an intety space, or indirectly after traversing the pleurat cavity and/or lung; across the oesophagus or from the duodenum into the vena cava ; across the dia phragm, from the abdominal wall or the per toneal cavity ; across the respiratory tract ; or bof transport in the bloodstream from a distant regiono

The commonest route is through the chest walb

Sundry aspects of injuries from pins and needle distinguish them from other varieties of cardiad trauma. The principal distinction concerns theif dimensions and configuration, the significance of 
which was apparently appreciated a few centuries ago. During the scientific renaissance, many people disproved Aristotle's dictum about cardiac inviolability by sticking pins and needles into the hearts of animals. They demonstrated that such wounding was remarkably well tolerated and compatible with life. Moreover, exsanguination did not happen, as had been supposed, except when less fleshly, non-sealing portions of the heart were pricked. These trials were made directly on the organ, and it was not until much later, when clinical accounts were published, that notable differences from the experimental setting became evident. Paradoxically, the most obvious of these differences has not been emphasized in the literature. It concerns the fact that the heart is more vulnerable to serious injury while the foreign body is extracardiac than when it is intracardiac.

It is clear that the major damage is caused when the object is partially immobilized-in the thoracic parietes, for example. Then, repetitive wounding results from the unceasing motion of the heart against the sharp point of the fixed foreign body. The danger to life of rigidly held objects is exhibited in the cases surveyed. Quite often the hearts have borne marks denoting continuous puncturing, scraping in saw-tooth pattern, or slicing along a semilunar or elliptical arc. Intact pins have been prime offenders in this connexion, since their broadened end has ordinarily restrained free passage into the thorax. In one particularly striking case, the heart shredded itself against a brooch-pin rendered immovable by an ornate handle. Of course, the longer the foreign body, the less likely is this mechanism to become operative, but then the greater are the risks of simultaneous skewering of several portions of the heart. This is especially true in younger subjects, in whom the easy compressibility and small size of the chest magnify the importance of even a $2-\mathrm{cm}$. needle.

The next feature of interest regarding this category of foreign body is that the sleek and narrow contour allows fairly swift migration into the heart. Because of incessant movements of the rib cage and cardiac contractions, needles and fractured pins caught by one end in the myocardium do not remain stationary in the soft tissues for long. They are pulled gradually, but inexorably, into the substance of the heart. By then the bulk of the damage has been done. Haemopericardium is the principle cause of complication and death, and is due mainly to rents on the surface of the heart rather than to the slender, self-obliterating path left by the object as it passes through the wall of the heart. If the object is delivered with great impact, it is more likely to be forced into the heart at once, so that the phase of scratching or cutting of the cardiac surface is circumvented and haemopericardium is minimal or absent. In fact, such trauma seems to be associated with a more favourable outcome. This remarkable process has been noticed on several occasions, especially when the object possesses free mobility. In some instances the steadily forward course into the heart has been visualized by means of successsive radiographs. In others, needles have been seen to move or have disappeared from sight prior to or at operation because of vigorous contractions of the heart. In one case the advance of a pin through the heart wall was followed on serial radiographs until the head of the object halted its further transit.

It is noteworthy that the progress of haemopericardium has been unpredictable, numerous patients dying many days or weeks after the traumatic incident. Necropsy descriptions make it probable that some of these late deaths from tamponade have been from a secondary haemopericardium subsequent to realignment of the object, accompanied by grazing or laceration of the heart from within outward. The evolution of this phenomenon depends in large part on the axis of entrance of the object and where inside the heart chambers it becomes situated. For instance, if a needle becomes embedded in a straight line in the ventricular septum, further travel would tend to be impeded. Conversly, were it not thus stabilized, but floated loose in the left atrium, it would be more likely to migrate, as do needles and pins in other parts of the body where there is much movement. In the series there are only three examples of a needle being extruded by the heart back into the pleural cavity, and thence to between the ribs.

Needles which become fixed in the substance of the heart may remain clinically harmless for a long time, as attested by their having been found incidentally at necropsy. The longest asymptomatic interval has been 37 years. These are exceptional situations, however. The inflammatory reaction provoked after the object has been encompassed eventually results in its becoming 'encysted'. On the other hand, this walling-off may be hindered by infection or by thrombosis. That a silent foreign body need not necessarily signify benign encapsulation is exemplified by a multiplicity of complications arising at a remote period after the initial trauma.

Both focal pericarditis and chronic constrictive pericarditis have been described from weeks to 
years following injury. At times they have ended in death. Often sepsis has featured prominently as the agency of morbidity or in hastening the patient's death. Clot has frequently been found attached to parts of pins and needless exposed to intracardiac blood currents. Death has ensued in some patients from embolization of fragments of such thrombi, especially the suppurative ones. A few of the objects have even become rusty or corroded from contact with blood.

TREATMENT The outcome of cardiac injuries due to pins and needles has been characterized in the past by a high mortality, which should serve as a warning against any misconception that these foreign bodies are innocuous. Death in most cases has been due to cardiac tamponade.

There is little quibbling about the necessity for prompt surgical intervention when an intracardiac foreign body of any sort produces symptoms or menaces life. Emergencies from pin and needle injuries are predominantly instigated by cardiac tamponade. Pericardiocentesis is permissibleindeed, probably desirable - as a prelude to an operation intended to retrieve the foreign body and repair whatever damage it has inflicted when haemopericardium is present. Undue temporizing in such situations is ill-advised, for, as a number of the reviewed cases have shown, death may follow quite quickly on the heels of demonstrable haemopericardium. Precise radiographic localization of the foreign body is desirable but is not essential. This information can be secured by films taken while the patient is in the operating room, with all necessary equipment at hand in the event of precipitous deterioration in his condition. Moreover, it sometimes happens that the object will have migrated by the time operation is begun. Once the foreign body has been identified on admission to hospital, the goal should be to remove it as soon as possible without delays entailed by additional non-contributory studies.

Pericardiotomy should suffice in most instances for evacuating the haemopericardium, withdrawing the object, and performing cardiorrhaphy. The exposure must be adequate since unsuccessful operations in the series have been associated with inadequate procedures or limited manœuvrability through 'key-hole' incisions. It is immaterial whether a vertical sternotomy or an anterolateral thoracotomy approach is selected, providing there is unhampered accessibility to all regions of the heart and great vessels. Ancillary aids, such as electromagnetic localizers, have not been useful in these cases.
Deliberate drainage of the pericardial sac, $\overrightarrow{\vec{s}}$ regardless of the presence or absence of haemo-o pericardium, should be accomplished always.을 Failure to do so, or tight re-suture of the peri- $\frac{\bar{c}}{\sqrt{5}}$ cardium, has been accompanied at times by the reaccumulation of pericardial fluid. In the samen context, even if a pin or needle is amenable tos simple removal, i.e., digitally or by incising the skin over it, the physician is not relieved of re $\overrightarrow{\vec{\omega}}$ sponsibility for protracted management of the case. Rather, the patient should be admitted to hospital and kept under physical and radiologica surveillance to forestall cardiac tamponade from delayed bleeding. In a not inconsiderable number $N$ of cases, the insidious progression of haemopericardium after the aforementioned simpleo manoeuvres, or fresh haemopericardium due to other unsuspected foreign bodies, have been over-z looked with fatal consequences.

Whereas there is no equivocation about the urgency for operating in acute symptomatic phases of cardiac wounding by foreign bodies? doubts exist in some quarters regarding proper criteria for surgically 'molesting' chronic intrao cardiac foreign bodies. There is no denying thas some pins and needles of long standing in the heart may not cause any demonstrable harm. Bug these are medical curiosities. The dangers of late embolic, inflammatory, and septic sequelae from $\overrightarrow{\vec{b}}$ retained pins and needles are real, and transcend $\mathrm{B}$ the risks from striving for surgical retrieval of these objects. While acknowledging that generalo izations in medicine are incautious, we neverthe less wish to express our conviction that alP intracardiac foreign bodies should be removed even if silent clinically. That is surely a reasonable course to adopt, for not only does it effectivel safeguard against the eventuality of organie disease, but it also dispels uneasiness or othe emotional stress which the patient is likely tof have about being the bearer of a potentially deadl object in the heart.

\section{REFERENCES} Bajardi, F. (1953). Ueber einen Fall von Pflanzenzellembolien inn
Koronargefässsystem. Wien. klin. Wschr., 65, 815.

Barrett, N. R. (1950). Foreign bodies in the cardiovascular system? Brit. J. Surg., 37, 416.

Bláha, J. (1935). Embolische Verschleppung eines Fremdkörpere (Gummikatheter) ins Herz bei einem kriminellen Abortus. $Z b{ }_{\mathbb{D}}$

Decker, H. R. (1939). Foreign bodies in the heart and pericardium should they be removed? J. thorac. Surg., 9, 62 .

Farber, S., and Craig, J. M. (1956). Clinical Pathological Conference Children's Medical Center, Boston, Mass. J. Pediat., 49, 330.

Gajdas, J. (1963). Rib fracture in a 4-year-old child with perforation of the lung and right cardiac atrium by an ossal sequester. PaCD pol., 14, 521. (In Polish : English summary.)

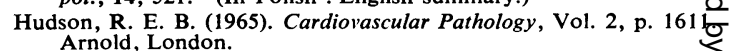


Leonard, J. W., and Gifford, R. W., Jr. (1965). Migration of a Kirschner wire from the clavicle into the pulmonary artery. Amer. J. Cardiol., 16, 598 .

Lillehei, C. W., Bonnabeau, R. C., Jr., and Grossling, S. (1965). Removal of iatrogenic foreign bodies within cardiac chambers and great vessels. Circulation, 32, 782 .

Lowen, H. J., Fink, S. A., and Helpern, M. (1950). Transfixion of the heart by embedded ice pick blade with 8 months' survival. Ibid., 2, 426 .
Parmley, L. F., Mattingly, T. W., and Manion, W. C. (1958). Penetrating wounds of the heart and aorta. Ibid., 17, 953.

Schiff, A. F. (1965). A fatality due to acupuncture. Med. Tms (N.Y.), 93,630 .

Tänzer, A (1953). Intrakardiale abgebrochene Injektionskanüle Fortschr. Röntgenstr., 78, 357.

Weekley, A. S., Jr., and Maltby, G. L. (1963). More stud-gun injuries (letters). New Engl. J. Med., 269, 110, 111.

Zacchias, P. (1600). Quaestiones medico-legales, I (5), 375. 